\title{
Hydrogenized Water Effects on Protection of Brain Cells from Oxidative Stress and Glutamate Toxicity
}

\author{
Robert Settineri $^{1, *}$, Jie Zhou ${ }^{2}$, Jin $\mathrm{Ji}^{3}$, Rita R. Ellithorpe ${ }^{4}$, Steven Rosenblatt ${ }^{5}$, Antonio Jimenez ${ }^{6}$, \\ Shigeo Ohta ${ }^{7}$, Gonzalo Ferreira ${ }^{8}$, Garth L. Nicolson" \\ ${ }^{1}$ Sierra Productions Research, Irvine, CA USA \\ ${ }^{2}$ Brunswick Laboratories, Inc., Southborough, MA USA \\ ${ }^{3}$ PulchriBio Intl, Boston, MA USA \\ ${ }^{4}$ Tustin Longevity Center, Tustin, CA USA \\ ${ }^{5}$ Saint John's Health Center, Santa Monica, CA USA \\ ${ }^{6}$ Hope4Cancer Institute, Baja California, Mexico \\ ${ }^{7}$ Department of Neurology, Juntendo University, Graduate School of Medicine, Tokyo, Japan \\ ${ }^{8}$ Department of Biophysicas, Laboratory of Ion Channels, School of Medicine, Universidad de la Republica, Montevideo, Uruguay \\ ${ }^{9}$ Department of Molecular Pathology, The Institute for Molecular Medicine, Huntington Beach, CA USA \\ *Corresponding author: sierraprod@aol.com
}

\begin{abstract}
Hydrogenized water is known to have protective effects on cells and tissues, mainly through its antioxidant activities. Here we examined the protective effects of a commercial source of hydrogenized water on cultured human brain cells. Hydrogenized water was able to protect brain cells from oxidative stress and glutamate toxicity. At $\mathrm{H}_{2}$ concentrations above $0.01 \mathrm{mM}$ the glutathione levels increased in cultured brain cells. The level of glutathione rose from approximately 500 to approximately $850 \mu \mathrm{M}$ at the maximum dose of hydrogenized water with an $\mathrm{EC}_{50}$ of approximately $0.030 \mathrm{mM}$. Hydrogenized water was also able to enhance the signaling pathway for oxidative stress response mediated by Nrf2 (Nuclear factor erythroid 2 like factor). Treatment of cells with hydrogenized water at concentrations above $0.01 \mathrm{mM} \mathrm{H}_{2}$ induced activation of $\mathrm{Nrf2}\left(\mathrm{EC}_{50}\right.$ approximately $\left.0.05 \mathrm{mM}\right)$. Hydrogenized water was also able to protect brain cells against glutamate toxicity. Using a DNA damage response element, $\left(\gamma \mathrm{H} 2 \mathrm{AX}\right.$, to monitor the damage of glutamate toxicity we found that concentrations of $\mathrm{H}_{2}$ above $0.01 \mathrm{mM}$ protected cells from glutamate damage with an $\mathrm{EC}_{50}$ of approximately $0.05 \mathrm{mM} \mathrm{H}_{2}$. These in vitro results demonstrated that hydrogenized water can protect brain cells against common types of damage from oxidative stress and glutamate toxicity.
\end{abstract}

Keywords: hydrogenized water, bioassays, glutathione, oxidative stress, Nrf2, glutamate toxicity

Cite This Article: Robert Settineri, Jie Zhou, Jin Ji, Rita R. Ellithorpe, Steven Rosenblatt, Antonio Jimenez, Shigeo Ohta, Gonzalo Ferreira, and Garth L. Nicolson, "Hydrogenized Water Effects on Protection of Brain Cells from Oxidative Stress and Glutamate Toxicity." American Journal of Food and Nutrition, vol. 6, no. 1 (2018): 9-13. doi: 10.12691/ajfn-6-1-2.

\section{Introduction}

Cells within the central nervous system (CNS) are among the most metabolically active cells in our bodies, and they are also among the most sensitive to oxidative stress $[1,2,3]$. Oxidative stress occurs when there is an excess of reactive oxygen and nitrogen species (ROS, RNS) over natural and added antioxidant molecules $[3,4,5,6,7]$. Under normal circumstances reactive oxidants, ROS and RNS, are produced in low amounts where they can act as important signaling molecules in cytoplasmic events, such as growth factor signaling, mitochondrial autophagy, and nuclear cell division. ROS and RNS are also important in immune function, inflammation and other important cellular and tissue responses $[3,4,5,6,7]$.

Excess oxidative stress in the brain is associated with and contributes to the etiology or progression of neurodegenerative diseases. This is due to the disproportionate amount of oxygen use in this tissue and the chance of excess production of ROS/RNS that can damage brain cell lipids, proteins and nucleic acids $[7,8,9]$. One of the systems that neutralizes excess oxidative stress due to ROS/RNS is the glutathione system [10]. Glutathione is a ubiquitous thiol that acts in concert with other antioxidants to reduce ROS/RNS and prevent damage to brain cells $[9,10,11,12]$. It is also a transfer molecule for cysteine and is involved in keeping sulfhydryl proteins in a reduced state, which is important in maintaining cellular redox potential as well as for detoxification $[10,12,13]$.

Another cellular defense system against oxidative stress in the brain is mediated by the nuclear factor erythroid 2-related factor (Nrf2) [14,15]. Nrf2 is a member of the cap'n'collar family of leucine zipper transcription factors that in the nucleus heterodimerizes with small proteins (MAF, JUN proteins) and binds to DNA sites containing anti-oxidant response elements (ARE) and other sites to 
initiate transcription of cytoprotective genes [16]. The Nrf2 system is also linked to activation of $\mathrm{NF \kappa B}$ to mediate anti-inflammatory effects $[17,18]$. Insufficient Nrf2 activation in humans has been linked to neurodegenerative diseases, such as Parkinson's disease, Alzheimer's disease and Amyotrophic Lateral Sclerosis $[17,19]$.

In addition to oxidative stress, there are several markers for toxicity in the brain, but one of the most widely studied is the neurotransmitter L-glutamate. L-glutamate is a major excitatory neurotransmitter in the brain, and it is involved in virtually all activities of the nervous system [20,21]. L-glutamate binds to and activates both ligand-gated ion channels (ionotropic glutamate receptors) and a class of $\mathrm{G}$ protein-coupled receptors (metabotropic glutamate receptors). The intracellular glutamate concentration in the brain is normally in the millimolar range; however, extracellular glutamate concentration is kept low (in the low micromolar range) by excitatory amino acid transporters that import this molecule into astrocytes and neurons. When extracellular glutamate is in excess, this can result in excitotoxicity as seen in ischemic stroke and neurodegenerative diseases [20,21].

Hydrogenized water has been shown to act as a cellular antioxidant and gene regulator [22,23]. Previously we found that hydrogenized water was an effective cellular antioxidant and could decrease transport of glucose in a dose- and time-dependent manner and elevate the levels of phosphorylation of Akt, a protein kinase B biomarker for insulin signaling [24]. We also found that hydrogenized water could increase the levels of age-related SIRT1 gene expression and increase the expression of telomerase activity in a dose-dependent process [24]. Here we examined the ability of hydrogenized water in vitro to protect brain cells from oxidative damage by increasing glutathione levels and reducing glutamate toxicity. We also examined the ability of hydrogenized water to activate the $\mathrm{Nrf} 2$ pathway as a marker for the antioxidative and anti-inflammatory activities.

\section{Materials and Methods}

Hydrogenized water identified as "IZUMIO Hydrogenized Water" was acquired from Naturally Plus USA (NPUSA) Las Vegas, Nevada. At the time of initial packaging, the infused hydrogen within the test water was analyzed by both manometric and diaphragm polarographic electrode measurements at final concentration of $2.6 \mathrm{ppm}(1.3 \mathrm{mM}$ $\mathrm{H}_{2}$ ) and $\mathrm{pH}$ 7.0. The Izumio hydrogenized water was used in all of the assays [24].

The human neuroblastoma SH-SY5Y cell line and HepG2 cell line were obtained from American Type Culture Collection (ATCC, Manassas, VA, USA). Dulbecco's modified Eagle's medium (DMEM), Eagle's Minimum Essential Medium (EMEM), and F12 Medium were purchased from GIBCO ${ }^{\mathrm{TM}}$ (Grand Island, NY, USA). Fetal bovine serum (FBS), Minimum Essential Media (MEM) and Earle's Balanced Salt Solution were purchased from Hyclone (Pittsburg, PA, USA). Sodium pyruvate, penicillin, streptomycin, quercetin, formaldehyde solution, Triton X-100 and cell culture grade water were obtained from Sigma (St. Louis, MO, USA). Dulbecco's phosphate-buffered saline (DPBS), Monochlorobimane, Hoechst 33342 (DNA stain), primary antibody cocktail consisting of mouse-anti-phospho-histone H2A.X and Hoechst 333342 and secondary antibody Cy3-donkeyanti-rabbit-IgG were purchased from Thermo Fisher Scientific (Fremont, CA, USA). Reagents used in Nrf2 luciferase assay were purchased from Promega. Cell plates were purchased from Corning Costar Corporation (Cambridge, MA, USA).

\subsection{Glutamate Toxicity Assay}

The effects of hydrogenized water on brain cell protection was studied using glutamate toxicity as the cellular biomarker [25]. Human neuroblastoma SH-SY5Y cells were cultivated in a 1:1 mixture of Eagle's Minimum Essential Medium and F12 Medium with fetal bovine serum at a final concentration of $10 \%$, and were seeded onto a 24-well cell culture plate and cultured for $24 \mathrm{hr}$ before addition of the test samples in cell culture media. In situations of hydrogenized water treatment, culture medium as dry powder was suspended and dissolved in freshly opened hydrogenized water. Different final concentrations of the hydrogenized water in cell culture grade water were used or at $\mathrm{v} / \mathrm{v}$ ratios of $1,0.5,0.25$, $0.125,0.062$ and 0.03125 of hydrogenized water to cell culture grade water). After preparation, the culture medium was sterile-filtered and used to treat neuronal cells immediately. All experiments were performed in triplicate.

The SH-SY5Y cells were exposed to normal culture media or culture media made with various concentrations of hydrogenized water for $24 \mathrm{hr}$, and then challenged with $250 \mu \mathrm{M}$ glutamate for $24 \mathrm{hr}$. After washing twice with culture media, the cells were fixed with $3.7 \%$ formaldehyde solution for 30 minutes. The fixed cells were then washed with DPBS twice on a Biotek plate washer and then treated with permeabilization solution composed of $0.5 \%$ (v/v) Triton X-100 for $10 \mathrm{~min}$. After $10 \mathrm{~min}$ treatment, the cells were washed with DPBS once and blocked for 30 minutes with blocking solution ( $1 \%$ bovine serum albumin in DPBS) prior to antibody staining. After incubation in the blocking solution, cells were washed again with DPBS once and incubated with a primary antibody cocktail consisting of mouse-anti-phospho-histone H2A.X and Hoechst 333342 at room temperature for $1 \mathrm{hr}$. After washing twice with DPBS on a Biotek plate washer, secondary antibody (Cy3-donkey-anti-rabbit- $\operatorname{IgG}$ ) was dispensed into each well and incubated continued at room temperature for another hr. After washing twice with DPBS on a Biotek plate washer, $200 \mu \mathrm{L}$ of DPBS was left in each well. The plate was then sealed and read on the ArrayScan VTi using a Compartmental Analysis Bioapplication. Data was processed and reported with the LIM system. Inhibition curves and $\mathrm{EC}_{50} \mathrm{~s}$ were generated via Graphpad's Prism.

\subsection{Glutathione (GSH) Level Assay}

The effects of hydrogen water on brain cell protection were studied using the intracellular glutathione level as the cellular biomarker [26]. Human neuroblastoma SH-SY5Y cells were cultivated in a 1:1 mixture of Eagle's Minimum Essential Medium and F12 Medium with fetal bovine 
serum at a final concentration of $10 \%$, seeded into a 96-well cell culture plate and cultured for $24 \mathrm{hr}$ before addition of the test compound in cell culture media. When media containing hydrogenized water was used, culture medium dry powder was suspended and dissolved in freshly opened hydrogenized water, and different final concentrations of the hydrogenized water in cell culture grade water were used at v/v 1.00, 0.50, 0.250, 0.0125, 0.00625 and 0.003125 of hydrogenized water to cell culture grade water, as previously used for other cell types [24]. Culture medium was then sterile-filtered and used to treat neuronal cells immediately. All experiments were performed in triplicate.

To test for response to oxidative stress cells were treated with the hydrogenized water media for $24 \mathrm{hr}$ and then exposed to $\mathrm{H}_{2} \mathrm{O}_{2}(300 \mu \mathrm{M})$ for $2 \mathrm{~h}$. Cells were then incubated in control or test hydrogenized water media at the concentrations stated above for $24 \mathrm{hr}$. After washing twice with culture media, the cells in each well were incubated with Monochlorobimane (final concentration of $50 \mu \mathrm{M})$ mixed with $30 \mu \mathrm{l}$ of $10 \mathrm{mg} / \mathrm{ml}$ Hoechst 33342 for $15 \mathrm{~min}$ at $37^{\circ} \mathrm{C}$. After washing twice with PBS, the plates were read on an arrayscan VTI using 2 channels and acquiring a $10 \mathrm{X}$ protocol for one field. Data were processed and reported with the LIM system. Inhibition curves and $\mathrm{EC}_{50} \mathrm{~S}$ were generated via Graphpad's Prism.

\subsection{Cellular Nrf2 Activation Assay}

The effects of hydrogen water on cellular Nrf2 pathway were also studied. [27]. HepG2 cells were cultured in Dulbecco's modified Eagle's medium (DMEM, HyClone, USA) supplemented with $10 \%$ fetal bovine serum (FBS, HyClone, USA) and antibiotics $(50 \mathrm{U} / \mathrm{mL}$ of penicillin and $50 \mu \mathrm{g} / \mathrm{mL}$ streptomycin) under an atmosphere of $5 \%$ $\mathrm{CO}_{2} 95 \%$ air at $37^{\circ} \mathrm{C}$. The cells were then seeded at a density of 35,000 cells per well into a clear-bottom 96-well plate containing $100 \mu \mathrm{l}$ of growth medium (MEM/EBSS (Hyclone), 10\% FBS, 1\% non-essential amino acids, $1 \mathrm{mM}$ Na-pyruvate, and 1\% Pen/Strep, and then incubated overnight at $37^{\circ} \mathrm{C}$ in a $\mathrm{CO}_{2}$ incubator. ARE reporter was transfected into cells the next day and incubated overnight at $37^{\circ} \mathrm{C}$ in the $\mathrm{CO}_{2}$ incubator. Cells were then treated with the test media or control media on the following day. In situations where hydrogenized water in culture media were used for treatment, culture medium dry powder was suspended and dissolved in freshly opened hydrogenized water at different concentrations of the hydrogenized water in cell culture grade water at $\mathrm{v} / \mathrm{v}$ of $1.00,0.50,0.250,0.0125,0.00625$ and 0.003125 of hydrogenized water to cell culture grade water. Culture media were sterile-filtered and used to treat cells immediately. After incubation for $18 \mathrm{~h}$, the cells were lysed with $20 \mu \mathrm{L}$ cell culture lysis buffer as before, and incubated with $100 \mu \mathrm{L}$ luciferase assay substrate. Luminescence was measured using a multimode plate reader

\subsection{Statistical Analyses}

Because of the small sample sizes a non-parametric test was used, the Kruskal-Wallis test. The Kruskal-Wallis test is the non-parametric alternative to a one-way ANOVA and does not assume normality of the residuals. The null hypothesis of this test is that all the medians are equal. If the Kruskal-Wallis test is significant, it indicates that at least two concentrations have significantly different medians. Statistical analysis was performed using $\mathrm{JMP}^{\circledR}$, version 13 (SAS Institute, Inc., Cary, NC) by the Cornell University Statistical Consulting Unit, Ithaca, NY [28].

\section{Results and Discussion}

\subsection{Glutamate Toxicity}

Glutamate is a major excitatory neurotransmitter in the central nervous system that is involved in brain functions including cognition, memory and learning [29]. It articulates the signaling network that regulates brain development, differentiation and functioning of synapses [30]. Whereas an optimal dose of glutamate is essential for normal brain physiology, low and high doses can trigger neurotoxic or excitotoxic cascades [31]. Glutamate is mediated mainly via two types of ionotropic glutamate receptors, the NMDA receptor and non-NMDA receptor [32].

In this experiment, we monitored DNA damage response $(\gamma \mathrm{H} 2 \mathrm{AX})$ to evaluate the effect of a test material (hydrogenized water) on protection of human brain cells from glutamate toxicity. We treated human SH-SY5Y neuroblastoma cells for $24 \mathrm{hr}$ with a series of concentrations of hydrogenized water in culture media. Above $\mathrm{H}_{2}$ concentrations of $0.01 \mathrm{mM} \mathrm{H}_{2}$, the cellular content of $\gamma \mathrm{H} 2 \mathrm{AX}$ was reduced. The results indicated that $\gamma \mathrm{H} 2 \mathrm{AX}$ was lowered by $50 \%$ when cells were treated with a concentration equal to $52.8 \%\left(0.030 \quad \mathrm{mM} \mathrm{H}_{2}\right)$ of hydrogenized water (significance in the Kruskal-Wallis test, $\chi=13.21, p=0.0215$ ) (Figure 1). These results indicate that hydrogenized water may contribute to the recovery of DNA damage from glutamate toxicity in brain cells.

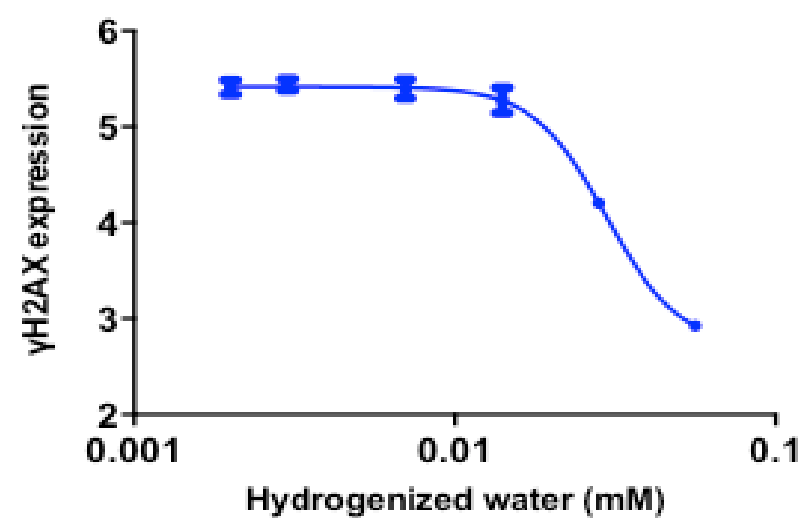

Figure 1. The effects of hydrogenized water on glutamate toxicity as monitored by DNA damage response $(\gamma \mathrm{H} 2 \mathrm{AX})$. Human SH-SY5Y neuroblastoma cells were treated with a series of culture media containing various concentrations of hydrogenized water for $24 \mathrm{hr}$ prior to exposure to glutamate. The protective effect of hydrogen against glutamate toxicity was determined by reductions in $\gamma \mathrm{H} 2 \mathrm{AX}$ as described in Materials and Methods. In this plot of the relative amounts of $\gamma \mathrm{H} 2 \mathrm{AX}$ (compared to an untreated control) versus hydrogen concentration the $\mathrm{EC}_{50}$ was calculated to be $0.030 \mathrm{mM} \mathrm{H}_{2}$

When excess extracellular glutamate is present, it can trigger excitotoxicity in vitro and in vivo in acute situations like ischemic stroke via over-activation of ionotropic 
glutamate receptors. In addition, chronic excitotoxicity has been found to play a role in several neurodegenerative diseases, including amyotrophic lateral sclerosis, Alzheimer's disease and Huntington's disease [33]. Thus maintenance of appropriate levels of extracellular glutamate is essential to prevent excitotoxicity in the central nervous system.

\subsection{Glutathione (GSH)}

Glutathione (GSH) is a key intracellular tripeptide thiol antioxidant that protects cells from free radical damage by providing reducing equivalents for the reduction of lipid hydroperoxides [9]. GSH depletion has been implicated in the pathogenesis of neurological diseases, particularly in Parkinson's disease (PD) [34,35]. In this assay, we evaluated the effectiveness of hydrogenized water in increasing the concentration of GSH in brain cells as a marker for antioxidant levels in neuronal cells [36]. This was done by treating human neuroblastoma SH-SY5Y cells for $24 \mathrm{hr}$ with a series of concentrations of hydrogenized water, then exposing the cells to oxidative stress and measuring the impact of hydrogenized water on GSH levels. The results indicate that GSH levels increased at $\mathrm{H}_{2}$ concentrations above $0.01 \mathrm{mM} \mathrm{H}_{2}$ or by $50 \%$ when cells were treated with $35 \%\left(0.0196 \mathrm{mM} \mathrm{H}_{2}\right)$ of hydrogenized water (significant difference in the Kruskal-Wallis test, $\chi=14.38, \mathrm{p}=0.0134$ ) (Figure 2). This indicates that hydrogenized water can contribute to the recovery of GSH levels during oxidative stress in brain cells.

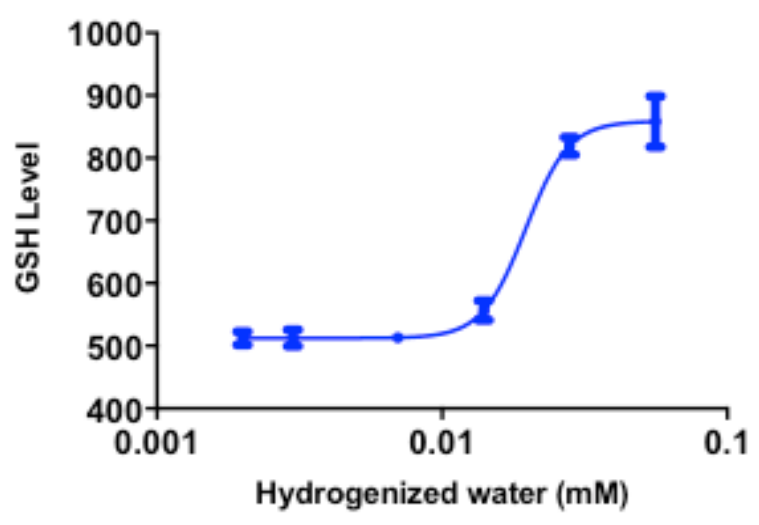

Figure 2. The effects of hydrogenized water on intracellular glutathione (GSH) levels in human SH-SY5Y neuroblastoma cells. The cells were treated with a series of culture media containing various concentrations of hydrogenized water for $24 \mathrm{hr}$ prior to exposing to oxidative stress challenge, and GSH levels were determined as described in Materials and Methods. In this plot of GSH level (compared to an untreated control) versus hydrogen concentration the $\mathrm{EC}_{50}$ was calculated to be $0.0196 \mathrm{mM} \mathrm{H}_{2}$

GSH participates in numerous cellular functions, and thus it is a critical cellular molecule $[35,36]$. GSH levels are correlated with increased oxidative damage and are known to diminish with increased age and in certain disease states [37,38]. Thus elevation in GSH levels protects cells from oxidative damage as well as damage due to toxic chemicals $[38,39]$.

\subsection{Cellular Nrf2 Activation}

Nuclear factor erythroid 2-related factor $2(\mathrm{Nrf} 2)$ is a redox-sensitive transcription factor that binds to antioxidant response elements (ARE) to regulate the expression of antioxidant enzymes that protect against oxidative damage triggered by injury and inflammation [40]. Activation of the Nrf2 pathway has been found to prevent and has been used to treat a large number of chronic inflammatory diseases [41]. A number of natural occurring phytonutrients, such as resveratrol, sulforaphane, and curcumin have been reported to activate $\mathrm{Nrf2}$, with additional phytonutrient $\mathrm{Nrf} 2$ activators being the focus of new investigations [42]. Nrf2 has also been investigated as a biomarker for regulating in vivo anti-oxidation and anti-inflammation responses [43].

In this experiment, we treated human cells with or without hydrogenized water, and the impact of the hydrogenized water on Nrf2 activation was determined. When hydrogenized water was added to the culture media at concentrations above $0.01 \mathrm{mM} \mathrm{H}_{2}$, the $\mathrm{Nrf} 2$ activation response was increased. We found that the level of $\mathrm{Nrf} 2$ activation increased by $50 \%$ when cells were treated with approximately $0.05 \mathrm{mM} \mathrm{H}_{2}$ (significance in the KruskalWallis test, $\chi=11.99, \mathrm{p}=0.035$ ) (Figure 3 ).

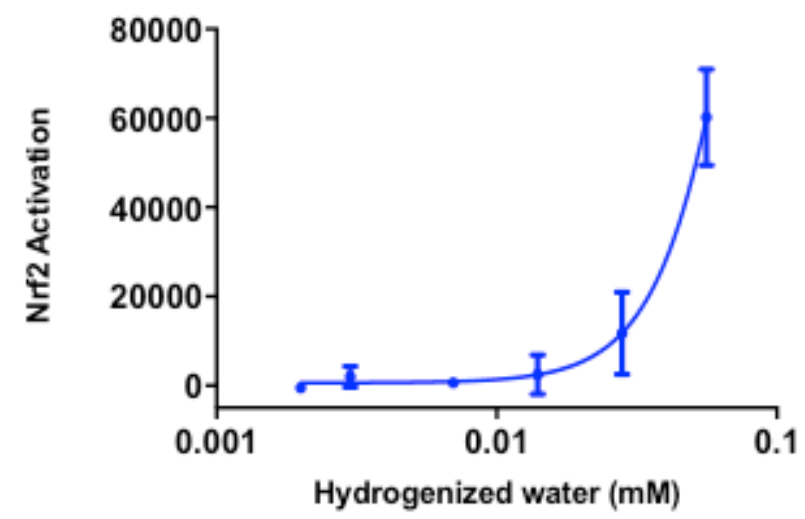

Figure 3. The effects of hydrogenized water on activation of transcription factor Nrf2 in human HepG2 cells. The cells were treated with a series of culture media containing various concentrations of hydrogenized water over night, and Nrf2 activation levels were determined as described in Materials and Methods. In this plot of relative Nrf2 activation level (compared to an untreated control) versus hydrogen concentration the $\mathrm{EC}_{50}$ was calculated to be $0.097 \mathrm{mM} \mathrm{H}_{2}$

Nrf2 is considered a multifunctional nuclear regulation factor [14,16,40,41]. As described above, it plays an important role as a cytoprotective factor regulating the expression of genes involved in anti-oxidant, antiinflammatory and detoxifying proteins, and it is also considered an important modulator of species longevity $[44,45]$. It is involved in the pathogenesis of some agedependent disorders, including neurodegenerative diseases. Thus substances like hydrogenized water that act to increase Nrf2 can play a stress-protective and anti-aging role and should be considered protective molecules against cellular damage and aging [45].

\section{Conclusion}

Molecular hydrogen has been shown to be useful in the treatment of various medical conditions and as an antiaging supplement [22,23]. Here we examined the effects of a commercial source of hydrogenized water on cultured human brain cells. Hydrogenized water protected brain cells from oxidative stress and glutamate toxicity. The commercial hydrogenized water also enhanced the signaling 
pathway for oxidative stress response mediated by Nrf2. These results demonstrated that hydrogenized water can protect brain cells against common types of cellular damage from oxidative stress and glutamate toxicity, important events in stroke and neurodegenerative diseases.

\section{Acknowledgement}

This work is supported by Naturally Plus USA.

\section{References}

[1] Beal MF. Mitochondria take center stage in aging and neurodegeneration. Ann Neurol. 2005; 58: 495-505.

[2] Dias V, Junn E, Mouradian MM. The role of oxidative stress in Parkinson's disease. J Parkinsons Dis. 2013; 3(4): 461-491.

[3] Schiavone S, Jaquet V, Trabace L, Krause K-H. Severe life stress and oxidative stress in the brain: from animal models to human pathology. Antioxid Redox Signal. 2013; 18(12): 1475-1490.

[4] Finkel T. Signal transduction by reactive oxygen species. J Cell Biol. 2011; 125: 376-393.

[5] Janssen-Heininger YM, Mossman BT, Heintz NH, Forman HJ, Kalyanaraman B, et al. Redox-based regulation of signal transduction: principles, pitfalls, and promises. Free Radic Biol Med. 2008; 45: 1-17.

[6] Navarro-Yepes J, Burns M, Anandhan A, Khalimonchuk O, Maria del Razo L, Quintanilla-Vega B, Pappa A, Panayiotidis MI, Franco R. Oxidative stress, redox signaling and authophagy: cell death versus survival. Antioxid Redox Signal. 2014; 21(1): 66-85.

[7] Fischer R, Maier O. Interrelation of oxidative stress and inflammation in neurodegenerative diseases: role of TNF. Oxid Med Cell Longev. 2015; 2015: 610813.

[8] Di Meo S, Reed TT, Venditti P, Victor VM. Role of ROS and RNS sources in physiological and pathological conditions. Oxid Med Cell Longev. 2016; 2016: 1245049.

[9] Smeyne M, Smeyne RJ. Glutathione metabolism and Parkinson's disease. Free Radical Biol Med. 2013; 62: 13-25.

[10] Meister A, Anderson ME. Glutathione. Annu Rev Biochem. 1983; 52: 711-760.

[11] Aoyama K, Nakaki T. Impaired glutathione synthesis in neurodegeneration. Int J Mol Sci. 2013; 14: 21021-21044.

[12] Johnson WM, Wilson-Deflosse AL, Mieyal JJ. Dysregulation of glutathione homeostasis in neurodegenerative diseases. Nutrients. 2012; 4: 1399-1440.

[13] Main PAE, Angley MT, O'Doherty CE,Thomas P, Fenech M. The potential role of the antioxidant and detoxification properties of glutathione in autism spectrum disorders: a systematic review and meta-analysis. Nutr Metab. (Lond) 2012; 9:36.

[14] Ma Q. Role of Nrf2 in oxidative stress and toxicity. Аnnu Rev Pharmacol Toxicol. 2013; 53: 301-426.

[15] Huang Y, Li W, Su Z-Y, Kong A-NT. The complexity of the Nrf2 pathway: beyond the antioxidant response. J Nutr Biochem. 2015; 26: 1401-1413.

[16] Tebay LE, Robertson H, Durant ST, Vitale SR, Penning TM, Dinkova-Kostova AT, Hayes JD. Mechanisms of activation of the transcription factor Nrf2 by redox stressor, nutrient cues, and energy status and the pathways through which it attenuates degenerative disease. Free Radical Biol Med. 2015; 88: 108-146.

[17] Sandberg M, Patil J, D'Angelo B, Weber SG, Mallard C. Nrf2regulation in brain health and disease: implication of cerebral inflammation. Neuropharmacol. 2014; 79: 298-306.

[18] Huang Y, Li W, Su Z-Y, Kong A-N T. The complexity of the Nrf2 pathway: beyond the antioxidant response. J Nur Biochem. 2105; 26: $1401-1413$

[19] Ramsey CP, Glass CA, Montgomery MB, Lindl KA, Ritson GP, Chia LA, et al. Expression of Nrf2 in neurodegenerative diseases. J Neuropathol Exp Neurol. 2007: 66: 75-85.

[20] Marmiroli P, Cavaletti G. The glutamatertic neurotransmission in the central nervous system. Curr Med Chem. 2012; 19: 1269-1276.

[21] Zhou Y, Danbolt NC. Glutamate as a neurotransmitter in the healthy brain. J Neural Transm. 2014; 121: 799-817.
[22] Ohta S. Molecular hydrogen as a novel antioxidant. Overview of the advantages of hydrogen for medical applications. Meth Enzymol. 2015; 555: 289-317.

[23] Nicolson GL, Ferreira de Mattos G, Settineri R, Costa C, Ellithorpe R, Rosenblatt S, La Valle J, Jimenez A, Ohta S. Clinical effects of hydrogen administration: from animal and human diseases to exercise medicine. Intern J Clin Med. 2016: 7: 32-76.

[24] Settineri R, Ji J, Luo C, Ellithorpe RR, Ferreira de Mattos G, Rosenblatt S, La Valle J, Jinenez A, Ohta S, Nicolson GL. Effects of hydrogenized water in intracellular biomarkers for antioxidants, glucose uptake, insulin signaling and SIRT1 and telomerase activity. Am J Food Nutrit. 2016; 4: 161-168.

[25] Navjot S, Singh R, Sarangi U, Saxena N, Chaudhary A, Kaur G, Kaul SC, Wadhwa R. Combinations of Ashwagandha leaf extracts lprotect brain-derived cells against oxidative stress and induced differentiation. PLos One. 2015; 10: e0120554.

[26] Barayuga SM, Pang X, Andres MA, Panee A, Bellinger FP. Methamphetamine Decreases Levels of Glutathione Peroxidases 1 and 4 in SH-SY5Y Neuronal Cells: Protective Effects of Selenium. Neurotoxicol. 2013; 37: 240-246.

[27] Lee J-M, Chan K, Kan YW, Johnson JA. Targeted disruption of Nrf2 causes regenerative immune-mediated hemolytic anemia. Proc Natl Acad Sci USA. 2004; 101: 9751-9756.

[28] R Core Team. A Language and Environment for Statistical Computing. R Foundation for Statistical Computing, Vienna, Austria, 2017. https://www.R-project.org.

[29] Zhou Y, Danbolt NC. Glutamate as a neurotransmitter in the healthy brain. J Neural Transm. 2014; 121: 799-817.

[30] Mayford M, Siegelbaum SA, Kandel ER. Synapses and memory storage. Cold Spring Har Perspect Biol. 2012; 4: a005751.

[31] Ikonomidou C, Turski L. Why did NMDA receptor antagonists fail clinical trials for stroke and traumatic brain injury? Lancet Neurol. 2002; 1: 383-386.

[32] Traynelis SF, Wollmuth LP, McBain CJ, Menniti FS, Vance KM, Ogden KK, et al. Glutamate receptor ion channels: structure, regulation and function. Pharmacol Rev. 2010; 62: 405-496.

[33] Lewerenz J, Maher P. Chronic glutamate toxicity in neurodegenerative diseases-What is the evidence? Front Neurosci. 2015; 9: 469.

[34] Pearce RK, Owen A, Daniel S, Jenner P, Marsden CD. Alterations in the distribution of glutathione in the substantia nigra in Parkinson's disease. J Neural Transm. 1997; 104: 661-667.

[35] Mischley LK, Conley KE, Shankland EG, Kavanagy TJ, Rosenfeld ME, Duda JE, White CC, Wilbur TK, De La Torre P, Padowski JM. Central nervous system uptake of intranasal glutathione in Parkinson's disease. NPJ Parkinson's Dis. 2016; 2: 16002.

[36] Sies H, Berndt C, Jones DP. Oxidative Stress. Annu Rev Biochem. 2017; 86: 715-748.

[37] Liu Y, Hyde AS, Simpson MA, Barycki JJ. Emerging regulatory paradigms in glutathione metabolism. Adv Cancer Res. 2014; 122: 69-101.

[38] Currais A, Maher P. Functional consequences of age-dependent changes in glutathione status in the brain. Antioxid Redox Signal. 2013; 19: 813-822.

[39] Towsend DM, Tew KD. The role of glutathione-S-transferase in anti-cancer drug resistance. Oncogene 2003; 22: 7369-7375.

[40] Suryakant KN, Khatri R, Jaiswal AK. Regulation of Nrf2 - An update. Free Radic Biol Med. 2014; 66.

[41] Gao B, Doan A, Hybertson BM. The clinical potential of influencing $\mathrm{Nrf} 2$ signaling in degenerative and immunological disorders. Clin Pharmacol. 2014; 6: 19-39.

[42] Su Z-Y, Shu L, Khor TO, Lee JH, Fuentes F, Kong A-N T. A perspective on dietary phytochemicals and cancer prevention: oxidative stress, Nrf2 and epigenomics. Top Curr Chem. 2013; 329: $133-162$.

[43] Ma Q, He X. Molecular basis of electrophilic and oxidative defense: promises and perils of Nrf2. Pharmacol Rev. 2012; 64: 1055-1081.

[44] Loboda A, Damulewicz, Pyza E, Jozkowicz A, Dulak J. Role of Nrf2/HO-1 system in development, oxidative stress response and diseases: an evolutionarily conserved mechanism. Cell Mol Life Sci. 2016; 73: 3221-3247.

[45] Zhang H, Davies KJA, Forman HJ. Oxidative stress response and Nrf2 signaling in aging. Free Radic Biol Med. 2015; 88: 314-336. 